\title{
A CHEAT-PROOF GAME-THEORETIC FRAMEWORK FOR COOPERATIVE PEER-TO-PEER VIDEO STREAMING
}

\author{
Yang Gao, Yan Chen and K. J. Ray Liu \\ Dept. ECE, University of Maryland, College Park. \\ E-mail: \{yanggao, yan, kjrliu\}@umd.edu
}

\begin{abstract}
Cooperative peer-to-peer (P2P) streaming model, which enables cooperation among peers with large intra-group bandwidths, has been shown as a promising approach for video streaming applications. However, due to the inherent conflict between the individual peer's utility and the social optimality, designing an effective cooperative P2P streaming system becomes challenging. In this work, we first formulate the interactions among group peers as a cooperative P2P streaming game, which takes into account the unique characteristics of video. Then, a cheat-proof strategy is proposed to enforce rational group peers to achieve the optimal social welfare cooperatively. In particular, we prove theoretically that the proposed strategy can enforce truth-telling as an equilibrium, satisfy individual rationality and guarantee the budget balance of the system in an average sense. Finally, simulation results are shown to verify the effectiveness of the proposed scheme.
\end{abstract}

Index Terms - P2P, cooperative streaming, mechanism design, cheat-proof, game theory.

\section{INTRODUCTION}

Recently, P2P service model has been viewed as a promising alternative to the traditional client-server service model for video streaming applications [1]. By enabling peers to act not only as clients downloading data from the network but also as servers uploading data for other peers, the P2P model can greatly reduce the workload placed on the server and therefore makes the large-scale video streaming possible.

Although P2P video streaming systems can achieve promising results, several drawbacks of such systems have been reported, such as introducing a large number of unnecessary traverse links within a provider's network [2] and causing a huge amount of cross Internet Service Provider (ISP) traffic [3]. Moreover, these systems are vulnerable to the well known "free-riding" phenomenon [4]. To address these drawbacks, the work in [5] proposed a cooperative P2P video streaming framework, in which geographically neighboring peers that had large intra-group bandwidths were regarded as group peers. Then, cooperation among group peers was achieved through an evolutionary game theoretic approach.
However, the approach in [5] cannot achieve the system-wide optimal performance due to its underlying evolutionary game model.

Therefore, it becomes very important to design a cooperative $\mathrm{P} 2 \mathrm{P}$ streaming system in which rational group peers cooperate with each other to achieve the system-wide optimal streaming performance. Mechanism design is an effective tool towards this end, which has been applied to many resource allocation problems in communication networks. For example, in [6], a mechanism design based approach has been proposed to coordinate users to access the unlicensed band in an efficient way. The work in [7] proposed a truthful auction to enable dynamic spectrum access in wireless networks.

However, all these existing approaches cannot be directly applied to cooperative $\mathrm{P} 2 \mathrm{P}$ video streaming, due to the following two reasons. First, for content-aware video applications, the unique characteristics of video must be explicitly considered. Second, in the cooperative P2P streaming problem, the gain is determined by the level of cooperation while group peers share the duty rather than resources in typical resource allocation problems. In this work, we propose a cheat-proof game-theoretic framework for the cooperative P2P streaming problem, which can enforce rational group peers acting cooperatively to achieve the system-wide optimal streaming performance. Moreover, our framework explicitly takes into account the unique characteristics of video.

The rest of the paper is organized as follows. In Section 2 , we introduce the system model and the utility function. Then, we present the problem formulation in Section 3. In Section 4, the specific design of a cheat-proof strategy and its properties are discussed. Finally, we show the simulation results in Section 5 and draw conclusions in Section 6.

\section{SYSTEM MODEL AND UTILITY FUNCTION}

\subsection{System Model}

We study a P2P network that consists of several groups of peers who want to view a real-time video streaming simultaneously. Peers within the same group cooperate with each other to download video data either directly from the server or from peers in other groups. Then, the downloaded video 
data is shared within the group in a P2P manner.

Consider a group of peers numbered $1,2, \ldots, N$. If we denote the download rates of these peers as $R_{1}, R_{2}, \ldots, R_{N}$, respectively, then the total download rate achieved by all peers can be represented as $R=\sum_{i=1}^{N} R_{i}$. We assume that the upload and download bandwidths within the group are larger than those cross groups. Moreover, peers are assumed to be rational in the sense that they will act to maximize their utilities.

\subsection{Utility Function}

Given the total download rate $R$ and peer $i$ 's download rate $R_{i}$, we can write the utility function of peer $i$ as

$$
U_{i}\left(R, R_{i}\right)=\lambda g_{i}(R)-c_{i}\left(R_{i}\right),
$$

where $g_{i}(R)$ is the gain, $c_{i}\left(R_{i}\right)$ is the cost and $\lambda$ is a parameter that controls the gain to cost ratio. Note that the intra-group sharing cost is negligible due to the large upload and download bandwidths within the group.

In this work, we assume that the cost is a linear function of the individual peer's download rate as follows.

$$
c_{i}\left(R_{i}\right)=C_{i} R_{i},
$$

where $C_{i}$ is the cost per unit download rate of peer $i$.

The gain $g_{i}(R)$ is determined by how much peer $i$ is satisfied with the received video. Therefore, it is desired to incorporate the unique characteristics of video when designing gain functions. In this work, we first design the gain as a function of the peak signal-to-noise ratio (PSNR) of the video as

$$
g_{i}(R)=\ln (P S N R) .
$$

Here, $\ln (\cdot)$ function is adopted due to the fact that a certain quality difference in the high PSNR region is less significant than that in the low PSNR region. Then, by adopting a twoparameter distortion-rate model of video [8] and applying the relationship between PSNR and distortion, we have

$$
U_{i}\left(R, R_{i}\right)=\lambda \ln (\gamma+\beta R)-C_{i} R_{i},
$$

where $\gamma$ and $\beta$ are two parameters determined by the video.

\section{COOPERATIVE P2P STREAMING GAME}

From the utility function, we can see that for any peer, the gain is determined by the total download rate of the group while the cost is a linear function of his/her download rate. Since peers are rational, they tend to contribute less and act selfishly as "free-riders", which leads to the failure of the cooperative P2P streaming system.

In this work, to stimulate cooperation among rational group peers, we formulate this problem as a cooperative $\mathrm{P} 2 \mathrm{P}$ streaming game. In the proposed game, players are the $N$ group peers. Each player has his/her own utility function defined in (1) and a maximum download rate constraint $R_{i}^{\max }$.
The video streaming is also assumed to have minimum and maximum source rate constraints, which are denoted as $R^{\text {min }}$ and $R^{\max }$ respectively. Moreover, we assume that the unit cost of each user is a random variable drawn according to a probability density function (PDF) $f_{i}\left(C_{i}\right)$. Although the PDFs are assumed to be public information, realizations of the unit cost $\left\{\tilde{C}_{1}, \tilde{C}_{2}, \ldots, \tilde{C}_{N}\right\}$ are held privately to each player respectively. We divide time into time frames. At the beginning of each time frame, after observing his/her own private information, the $i$-th player will report a value $\hat{C}_{i}$ to the server, which is not necessarily the same as the true value $\tilde{C}_{i}$. Once received reports from all players, the server will decide the download rates of all players for this time frame, notify every player his/her assigned download rate and coordinate the video streaming according to such rate assignment.

Several optimality criteria, such as proportional fairness and Pareto optimality, can be adopted by the server to determine the optimal rate assignment. In this work, we choose to maximize the social welfare, which is the sum utility of all players. The problem can be mathematically expressed as

$$
\begin{array}{ll}
\max _{R_{1}, R_{2} \ldots, R_{N}} & \sum_{i=1}^{N}\left[\lambda \ln \left(\gamma+\beta \sum_{i=1}^{N} R_{i}\right)-\hat{C}_{i} R_{i}\right], \\
\text { subject to } & 0 \leq R_{i} \leq R_{i}^{\text {max }}, i=1,2, \ldots, N, \\
& R^{\text {min }} \leq R \leq R^{\text {max }} .
\end{array}
$$

By solving (2), we can obtain the optimal download rate assignment rule, which we show in the following proposition.

Proposition 1. Based on the reports of group peers, the server assigns the download rate for each peer according to the following rule.

$$
R_{i}^{*}=r_{i}\left(\hat{C}_{1}, \hat{C}_{2}, \ldots, \hat{C}_{N}\right)= \begin{cases}R_{i}^{\max } & \text { if } \hat{C}_{i}<C_{t h} \\ R_{t h} & \text { if } \hat{C}_{i}=C_{t h} \\ 0 & \text { if } \hat{C}_{i}>C_{t h}\end{cases}
$$

where $C_{t h}$ is a threshold determined by the Karush-KuhnTucker (KKT) conditions [9] of (2),

$R_{t h}=\frac{1}{\sum_{k=1}^{N} 1\left(\hat{C}_{k}=C_{t h}\right)}\left(R^{\text {total }}-\sum_{k=1}^{N} 1\left(\hat{C}_{k}<C_{t h}\right) R_{k}^{\max }\right)$, and $R^{\text {total }}=\min \left(R^{\max }, \max \left(R^{\min }, \frac{N \lambda}{C_{t h}}-\frac{\gamma}{\beta}\right)\right)$.

Proof: Due to page limitation, we show the proof in the supplementary information [10].

Since the rate assignment rule in (3) is the solution to (2), the social welfare of the entire group will be maximized if all peers report their true values, i.e. if $\hat{C}_{i}=\tilde{C}_{i}$ for all $i=$ $1,2, \ldots, N$. However, as the rate assignment rule in (3) always favors group peers that have high download costs, peers will tend to report larger values to the server to avoid the duty of downloading. Therefore, in order to achieve the system-wide 


$$
\begin{gathered}
\psi_{i}\left(\hat{C}_{i}\right)=E\left\{\sum_{j=1, j \neq i}^{N}\left[\lambda \ln \left(\gamma+\beta \sum_{k=1}^{N} r_{k}\left(C_{1}, C_{2}, \ldots, C_{N}\right)\right)-C_{j} r_{j}\left(C_{1}, C_{2}, \ldots, C_{N}\right)\right] \mid C_{i}=\hat{C}_{i}\right\} \\
\tilde{U}_{i}\left(\tilde{C}_{i}, \hat{C}_{1}, \hat{C}_{2}, \ldots, \hat{C}_{N}\right)=\lambda \ln \left(\gamma+\beta \sum_{k=1}^{N} r_{k}\left(\hat{C}_{1}, \hat{C}_{2}, \ldots, \hat{C}_{N}\right)\right)-\tilde{C}_{i} r_{i}\left(\hat{C}_{1}, \hat{C}_{2}, \ldots, \hat{C}_{N}\right)
\end{gathered}
$$

optimal streaming performance, the following question need to be addressed: how to provide incentives for rational group peers to report their true values.

\section{CHEAT-PROOF STRATEGIES}

In this section, we propose a mechanism design based approach to enforce truth-telling among rational group peers. In particular, every group peer will receive a transfer at the end of each time frame, which is measured by some virtual currency and can be either positive or negative. When the transfer of a player is positive, he/she gets compensation from the sever. On the other hand, the player will have to pay to the server if the transfer is negative. After introducing transfer functions, the overall utility function of a player becomes his/her original utility in (1) plus the transfer.

In the proposed cheat-proof strategy, we define the transfer function of the $i$-th player as

$t_{i}\left(\hat{C}_{1}, \hat{C}_{2}, \ldots, \hat{C}_{N}\right)=\sum_{j=1, j \neq i}^{N} U_{j}\left(R^{*}, R_{j}^{*}\right)-\frac{1}{N-1} \sum_{j=1, j \neq i}^{N} \psi_{j}\left(\hat{C}_{j}\right)$,

where $R^{*}=\sum_{i=1}^{N} R_{i}^{*}, R_{i}^{*}$ and $\psi_{i}\left(\hat{C}_{i}\right)$ are defined in (3) and (5), respectively. Note that the expectation in (5) is taken over the download costs of all players except player $i$.

Theorem 1. With the transfer functions in (4), the proposed mechanism is cheat-proof, i.e. it is an equilibrium that every player claims his/her true value.

Proof: It suffices to show that it is the best response of a player to report his/her true value when other players claim their true values. Without loss of generality, we assume players 2 to $N$ report the truth. Then, the necessary and sufficient condition of truth-telling being equilibrium can be written as $\forall \hat{C}_{1} \geq 0$,

$$
\begin{aligned}
& \tilde{U}_{1}\left(\tilde{C}_{1}, \tilde{C}_{1}, \tilde{C}_{2}, \ldots, \tilde{C}_{N}\right)+t_{1}\left(\tilde{C}_{1}, \tilde{C}_{2}, \ldots, \tilde{C}_{N}\right) \geq \\
& \tilde{U}_{1}\left(\tilde{C}_{1}, \hat{C}_{1}, \tilde{C}_{2}, \ldots, \tilde{C}_{N}\right)+t_{1}\left(\hat{C}_{1}, \tilde{C}_{2}, \ldots, \tilde{C}_{N}\right),
\end{aligned}
$$

where $\tilde{U}_{1}\left(\tilde{C}_{1}, \hat{C}_{1}, \hat{C}_{2}, \ldots, \hat{C}_{N}\right)$ is defined (7). Substituting (4) into (6) and eliminating common terms from both sides, we have $\forall \hat{C}_{1} \geq 0$,

$$
\begin{aligned}
& \tilde{U}_{1}\left(\tilde{C}_{1}, \tilde{C}_{1}, \tilde{C}_{2}, \ldots, \tilde{C}_{N}\right)+\sum_{i=2}^{N} \tilde{U}_{i}\left(\tilde{C}_{i}, \tilde{C}_{1}, \tilde{C}_{2}, \ldots, \tilde{C}_{N}\right) \geq \\
& \tilde{U}_{1}\left(\tilde{C}_{1}, \hat{C}_{1}, \tilde{C}_{2}, \ldots, \tilde{C}_{N}\right)+\sum_{i=2}^{N} \tilde{U}_{i}\left(\tilde{C}_{i}, \hat{C}_{1}, \tilde{C}_{2}, \ldots, \tilde{C}_{N}\right) .
\end{aligned}
$$

Both sides of (8) are the sum utility of all players with player 1 reporting different values. Moreover, since reporting a false value $\hat{C}_{1}$ only affects the assigned download rates and $\left\{R_{i}^{*}=r_{i}\left(\tilde{C}_{1}, \tilde{C}_{2}, \ldots, \tilde{C}_{N}\right)\right\}$ with $i=1,2, \ldots, N$ maximize the sum utility of all group peers, (8) holds for all $\hat{C}_{1} \geq 0$, which establishes Theorem 1.

From Theorem 1, we can see that with the proposed transfer functions, we successfully align each individual player's interest with the social objective and therefore enforce truthtelling as an equilibrium. Moreover, to make sure that players have incentives to participate in the proposed game, we need to guarantee that their overall utilities are greater than zero, which is called "individual rationality (IR)" in mechanism design theory [11]. Our results are summarized in the following theorem.

Theorem 2. When $\lambda \geq \max _{i=1,2, \ldots N}\left(\frac{E\left[C_{i}\right] R_{i}^{\max }}{\operatorname{In}\left(\gamma+\beta R^{\min }\right)}\right)$, the proposed cheat-proof strategy satisfies the individual rationality condition at the equilibrium, which can be mathematically expressed as follows.

$E\left[\tilde{U}_{i}\left(C_{1}, C_{2}, \ldots, C_{N}\right)+t_{i}\left(C_{1}, C_{2}, \ldots, C_{N}\right)\right] \geq 0, \forall i=1,2, \ldots, N$.

Proof: Due to page limitation, we show the proof in the supplementary information [10].

Moreover, the proposed transfer functions can guarantee budget balance of the system on average, which is shown in the following theorem.

Theorem 3. With the proposed cheat-proof strategy, the system can achieve budget balance as follows.

$$
E\left[\sum_{i=1}^{N} t_{i}\left(C_{1}, C_{2}, \ldots, C_{N}\right)\right]=0 .
$$

Proof: Due to page limitation, we show the proof in the supplementary information [10].

From Theorem 3, we can see that if the system operates for a relatively long time, the monetary value will only circulate within the group. Therefore, no extra monetary value is made from group peers by the server nor the server has to 


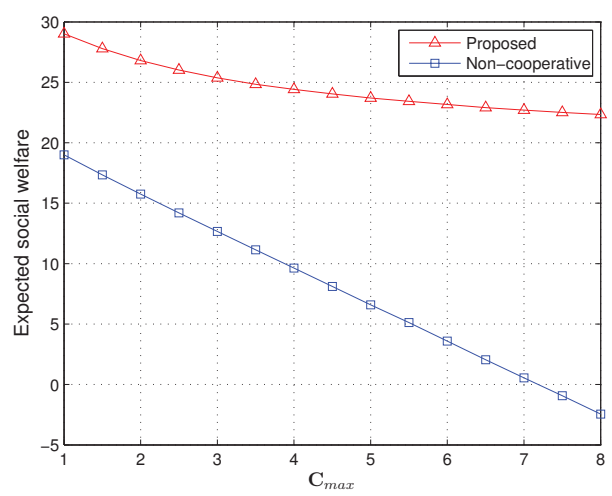

Fig. 1. Expected social welfare for the proposed framework vs. non-cooperative method.

pay group peers, which is a desired property especially for the $\mathrm{P} 2 \mathrm{P}$ streaming scenario. Note that the Vickrey-ClarkGroves (VCG) mechanism [12] can also be used to enforce truth-telling. However, the VCG mechanism cannot guarantee budget balance and therefore is less appropriate than the proposed cheat-proof strategy for the cooperative P2P streaming game.

\section{SIMULATION RESULTS}

In this section, we conduct numerical simulations to evaluate the proposed framework. A group of 6 peers is considered and the maximum rate constraint $R_{i}^{\max }$ is set as 3 for all peers. Moreover, we assume $\lambda=2, \gamma=5, \beta=1, R^{\text {min }}=1$ and $R^{\max }=10$ in the simulations.

We first show social welfare comparison between the proposed framework and the non-cooperative $\mathrm{P} 2 \mathrm{P}$ streaming method, in which peers act as individuals and maximize their own utilities without cooperation. Moreover, the unit cost of all peers are assumed to be i.i.d. random variables with uniform distribution on $\left[0, C_{\max }\right]$. We show the curves of the expected social welfare versus $C_{\max }$ for both methods in Fig. 1. From the figure, we can see that the proposed framework achieves much higher expected social welfare than the non-cooperative method. This is due to that the proposed framework enables peers to cooperate with each other in a way that the optimal social welfare is achieved.

Next, we evaluate the cheat-proof property of the proposed framework. $C_{\max }$ is set as 2 in this simulation. Moreover, we assume that all players report the truth except one particular player. Then, we evaluate the expected overall $\mathrm{u}$ tilities of this particular player versus his/her claimed values. The simulation results with three different true values are shown in Fig. 2. We can see that for all the three different true values, the player's expected overall utility is maximized when he/she truthfully reports the cost. Therefore, truth-telling is enforced by the proposed framework.

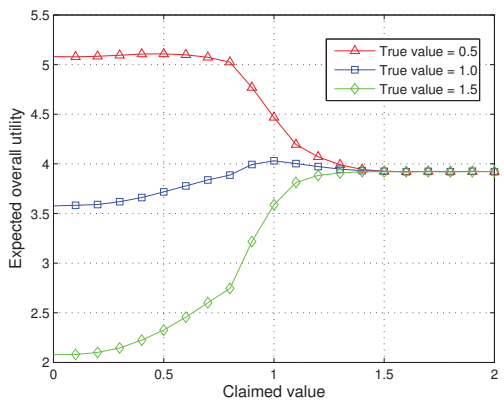

Fig. 2. The expected overall utilities versus the different claimed values.

\section{CONCLUSION}

In this paper, we propose a cheat-proof game-theoretic framework for cooperative $\mathrm{P} 2 \mathrm{P}$ streaming problems, which takes into account the unique characteristics of video. We prove theoretically and demonstrate though simulations that with the proposed framework, group peers get the incentive to truthfully reveal their private information and therefore can act cooperatively to achieve the optimal social welfare. Moreover, the proposed scheme guarantees both individual rationality and budget balance.

\section{REFERENCES}

[1] X. Zhang, J. Liu, B. Li, and T.-S. P. Yum, "Coolstreaming: A datadriven overlay network for peer-to-peer live media streaming," in IEEE Conference on Computer Communications (INFOCOM), pp. 2102-2111, 2005.

[2] H. Xie, Y. R. Yang, A. Krishnamurthy, Y. Liu, and A. Siberschatz, "P4P Provider portal for applications," in Proc. ACM SIGCOMM, 2008, pp. 351-362.

[3] T. Karagiannis, P. Rodriguez, and K. Papagiannaki, "Should internet service providers fear peer-assisted content distribution?," in Proceedings of the Internet Measurement Conference, 2005.

[4] M. Feldman, J. Chuang, "Overcoming free-riding behavior in peer-to-peer systems," SIGecom Exch., 5 (4) : 41-50, 2005.

[5] Y. Chen, B. Wang, W.S. Lin, Y. Wu, and K. J. R. Liu, "Cooperative peer-to-peer streaming: An evolutionary game-theoretic approach," IEEE Trans. on Circuits and Systems for Video Technology, vol. 20, no. 10, pp.1346-1357, Oct. 2010.

[6] Y. Wu, B. Wang, K. J. R. Liu, and T. C. Clancy, "Repeated open spectrum sharing game with cheat-proof strategies," IEEE Trans. Wireless Commun., vol. 8 , no. 4, pp. 1922-1933, Apr. 2009.

[7] X. Zhou, S. Gandhi, S. Suri, and H. Zheng, "eBay in the sky: Strategy-proof wireless spectrum auctions," in Proc. of ACM MobiCom, 2008.

[8] Y. Chen, B. Wang, and K. J. R. Liu, "Multi-user rate allocation games for multimedia communications," IEEE Trans. Commun., vol. 11, no. 6, pp. 1170-1181, Oct. 2009.

[9] S. Boyd, L. Vandenberghe, Convex Optimization, Cambridge Univ. Press, Cambridge, U.K., 2004.

[10] Supplementary information. [Online]. Available: http://www.sig. umd. edu/yanggao/SI.pdf

[11] D. Fudenburg and J. Tirole, Game Theory. MIT Press, 1993.

[12] W. Vickrey, "Counterspeculation, auctions, and competitive sealed tenders," $J$ of Finance, vol. 16, no. 1, pp. 8-37, Mar. 1961. 